\title{
Dynamic Response to Global Oscillation of Propulsion Systems with Cavitating Pumps
}

\author{
Shusuke Hori* \\ Japan Aerospace Exploration Agency, Tsukuba 305-8505, Japan \\ and \\ Christopher E. Brennen $\ddagger$ \\ California Institute of Technology, Pasadena, California 91125
}

DOI: $\underline{10.2514 / 1.51945}$

\begin{abstract}
A time-domain model was developed to evaluate the dynamic response of pumping systems in the accelerating environment of rockets with a focus on cavitation. The model was first verified by comparing the results with measurements in ground-based tests of an LE-7A rocket engine. In these tests, various resonances occurred and levels of pump cavitation or incorporation of an accumulator altered them. The model results simulated the test data well, matching both the frequency and the amplitude. The test and model results also demonstrated the stability of the LE-7A propulsion system within nonaccelerating environments. Then, the model was used to examine the response of the propulsion system in accelerating frames; sinusoidal vehicle oscillations over a range of frequencies were explored. Under noncavitating conditions, the pressure amplitudes within the propulsion system did not substantially exceed the quasi-static acceleration head response $\rho a h$. However, under cavitating conditions $(\sigma=0.02)$, the same accelerations produced violent responses with pressure and flow amplitudes about 2 orders of magnitude greater than in noncavitating conditions. The obvious conclusion is that vehicle oscillations can cause substantial pressure and flow amplitudes, particularly when the pump is cavitating, even if the ground-based tests and the calculations in static frames indicate stable and well-behaved responses.
\end{abstract}

\section{Nomenclature}

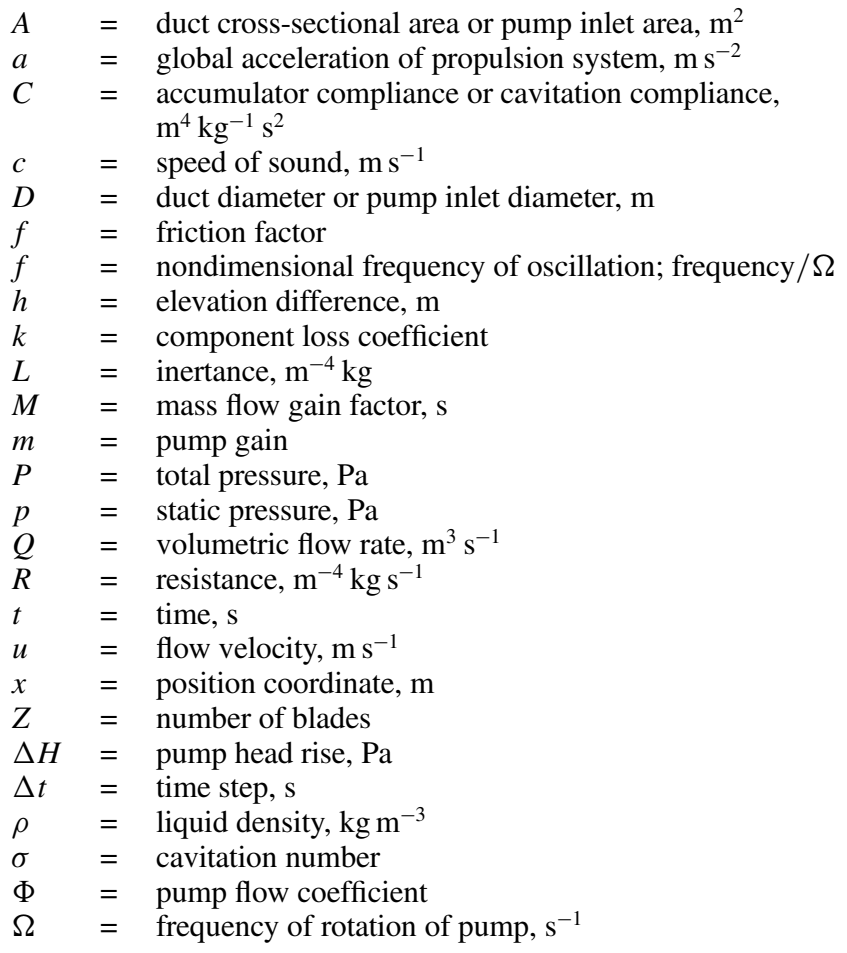

Received 9 August 2010; revision received 7 February 2011; accepted for publication 10 April 2011. Copyright @ 2011 by the American Institute of Aeronautics and Astronautics, Inc. All rights reserved. Copies of this paper may be made for personal or internal use, on condition that the copier pay the $\$ 10.00$ per-copy fee to the Copyright Clearance Center, Inc., 222 Rosewood Drive, Danvers, MA 01923; include the code 0022-4650/11 and \$10.00 in correspondence with the CCC.

*Staff Member, Space Transportation Mission Directorate, 2-1-1 Sengen; Visiting Associate, Department of Mechanical Engineering, California Institute of Technology, Pasadena, California 91125.

${ }^{\dagger}$ Professor, Department of Mechanical Engineering.

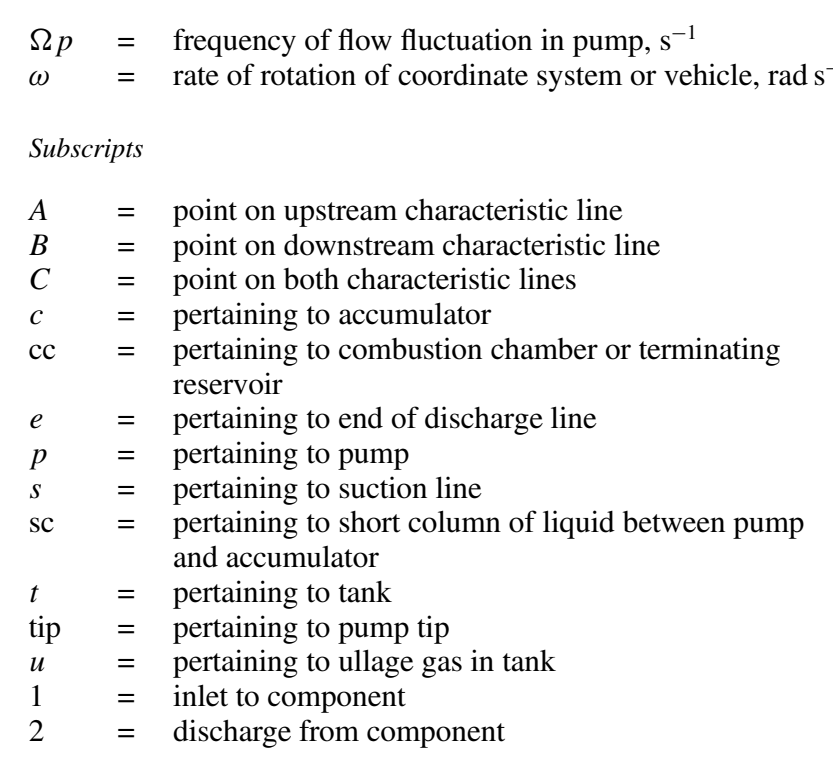

\section{Introduction}

W HEN a pumping system is subjected to shaking, the flow rates and pressures within that system can reach levels that may threaten the integrity of the system or radically alter its performance [1-3] . This could occur as a result of shaking imposed on a groundbased system or of an instability of a vehicle propulsion system of which the pumping system is a part [4,5]. The analysis by Rubin [4] of the pogo instability of a liquid-propelled rocket was an early example of a methodical approach to the dynamics of vehicle propulsion systems [5-7]. Since then, much research has been conducted to determine the dynamic response of rocket engine turbopumps [ 8 14], and the same kind of dynamic analysis has been used in other pumping contexts: for example, for primary coolant systems in power plants [15].

Virtually all of the experimental work on the dynamics of pumps has been necessarily conducted in ground-based test facilities (see, for example, $[\underline{10}-\underline{12}, \underline{14}])$, and much attention has therefore focused 
on the instabilities that are observed in pumping systems in a nonaccelerating environment (for example, [16-21]). Among these much-studied instabilities are those caused by cavitation in the pump: for example, rotating cavitation [18-21] and cavitation surge $[16,20]$. This last is perhaps the most violent and dangerous of these instabilities and, for the sake of the later discussion, it is worth briefly reviewing the simplest model of that phenomenon in the context of a cavitating inducer. The dynamics of a cavitating inducer can be represented as shown on Fig. 1 . In this model, one can visualize the lower path containing the resistance $R_{p}$ and the inertance $L_{p}$ as representing the main flow through the inducer, while visualizing the upper path containing the compliance $C_{p}$ and the mass flow gain factor $M_{p}$ as representing the cavitation and the backflow. Clearly, the natural frequency of the internal flow loop within the pump (consisting of the main flow and the backflow) will be given simply by

$$
\Omega_{p}=\frac{\left(L_{p} C_{p}\right)^{-1 / 2}}{2 \pi}
$$

Note that the resistance of the pump is positive when operating in a condition in which the head decreases as the flow increases, and this prevents any self-oscillation of the pump under conditions of little cavitation. However, as the cavitation number decreases, the compliance and the mass flow gain factor increase, and it is the latter that can feed energy into any instability.

As an example, we can point to the experimental measurements on a scale model of the space shuttle main engine (SSME) low pressure oxidizer turbopump $[\underline{1}, \underline{10}, \underline{11}]$, which indicate that appropriate values of the inertance and compliance in that case are given approximately by

$$
L_{p}=\frac{20}{D_{p}} \quad \text { and } \quad C_{p}=\frac{0.025 D_{p}}{\sigma(2 \pi \Omega)^{2}}
$$

where $\sigma$ is the cavitation number. Substituting the preceding approximate expressions into Eq. (1) yields

$$
\frac{\Omega_{p}}{\Omega}=(2 \sigma)^{1 / 2}
$$

which is precisely the frequency of cavitation surge put forward as an empirical observation in $[1,16]$ and reproduced in Fig. 2.

Returning to the general issue, less attention has been paid to the dynamic response of a pumping system (whether with or without cavitation), which is subjected to overall shaking, although the interaction of a launch vehicle's propulsion system and structure can be a source of dynamic instability (pogo). Since the days of the Gemini program, efforts have been made to find better ways to model and mitigate this potentially dangerous phenomenon [7]. In this paper, we explore the dynamic response of a pumping system to an imposed global oscillation and identify issues that should be of concern because, under global oscillation, they manifest substantial flow rate and pressure fluctuations in systems that are stable and benign in a nonaccelerating frame.

\section{Outline of Model}

A schematic of the dynamics of the type of system considered in this paper is sketched in Fig. 3; for example, this might represent the pumping system aboard a liquid-propelled rocket $[\underline{6}, 7]$. While the entire dynamical system would involve feedback through the response of the structure to fluctuating thrust, we focus here on the transfer function $G_{\text {hydraulic }}$ of the hydraulic propulsion system and its

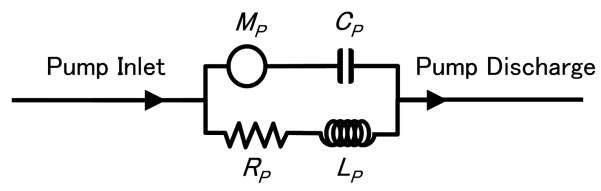

Fig. 1 Dynamic model of a cavitating pump.

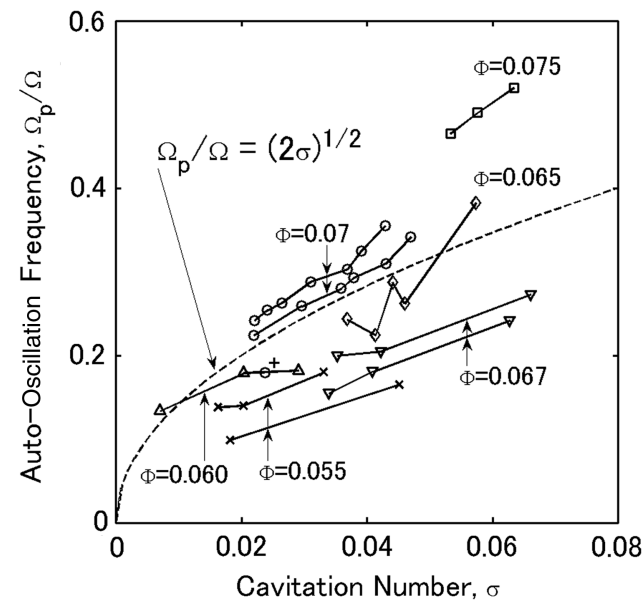

Fig. 2 Frequencies of cavitation surge from [1,16]: solid lines represent experimental observations of frequencies observed to occur for a number of different inducers operating at different flow coefficients $\Phi$, and the dotted line represents an empirical fit.

response to an imposed acceleration $a(x, t)$. In the complete system, the output from the hydraulic system would be a fluctuating thrust that would feed back into the structure to produce the acceleration $a(x, t)$ [4]. In the most general case, the structural oscillation and acceleration would be a function of both time $t$ and the position $x$ within the structure. However, for the purpose of simplicity, the results presented in this paper will be restricted to cases in which it is sufficient to simplify the issue by assuming a uniform acceleration $a(t)$, which is independent of position.

We begin by describing the time-domain model for the hydraulic pumping system, which incorporates the response to the imposed acceleration $a(t)$. The model connects the pressure $p(x, t)$ and flow rate $Q(x, t)$ [or volumetric velocity, $u(x, t)=Q(x, t) / A(x)$, where $A(x)$ is the cross-sectional area of the feedlines] at every location within the pumping system. While it is also possible to construct a frequency-domain model, we chose a time-domain approach in order to incorporate nonlinear inertial and frictional terms in the feedlines by the standard method of characteristics [1]. In addition, this leaves open the possibility of future incorporation of nonlinear effects in the components, particularly in the cavitating pump. These feedline models are married to a dynamic response model for the cavitating pumps that includes cavitation compliance and mass flow gain factor terms, as well as the known steady pump performance characteristic.

The methodology is designed to be applied to various configurations of pumping and propulsion systems, such as those sketched in Fig. 4. The examples include four different configurations pertaining to the turbopumps for the Japanese LE-7A rocket engine. Three of these configurations are ground-based facilities used to test designs of the turbopumps. The first two are cold-test facilities incorporating a turbopump: the first without a suction line accumulator and the second with a suction line accumulator. The third is a hot-firing engine test facility. The fourth configuration is the flight hardware consisting of the hydraulic system mounted in the accelerating rocket. Specific elements in these systems include the storage tank (fuel or oxidizer), compressible flow in the feedlines, an accumulator, valves, the turbopump, and discharge into a combustion chamber or catchment tank.

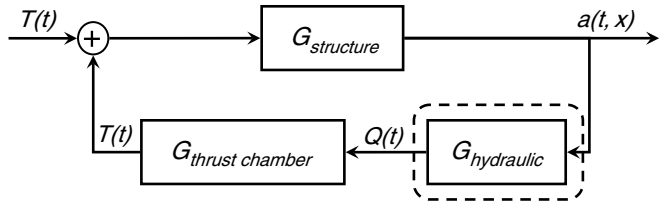

Fig. 3 Schematic of rocket propulsion system and dynamics: current paper focuses on response of hydraulic system (within dashed outline) to imposed oscillations. 


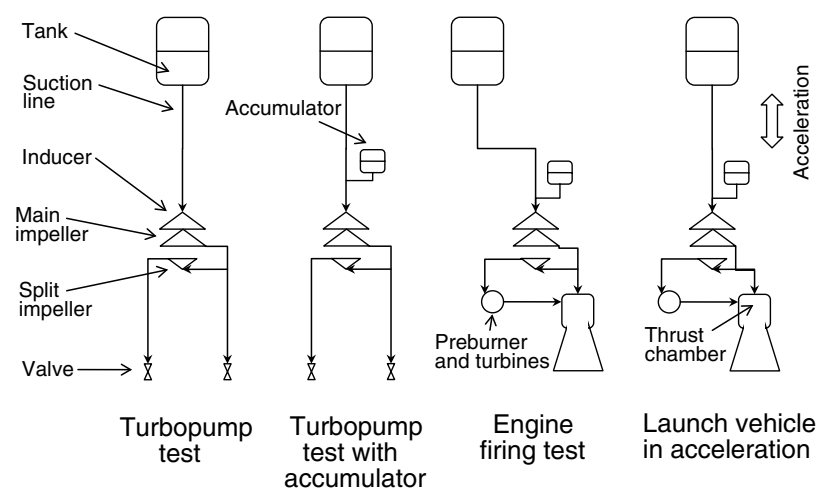

Fig. 4 Four hydraulic system configurations for which the dynamic responses are compared.

The assumed boundary conditions at inlet to and discharge from these hydraulic systems are an assumed storage tank pressure and the backpressure in the combustion chamber or catchment tank. It is convenient to choose the origin of the position vector $x$ to be the storage tank junction with the feedline; that coordinate and the acceleration $a$ are considered positive in the direction of flow. Consequently, if in the fourth configuration the vehicle is accelerating upward, the acceleration $a$ is negative.

\section{Details of Model}

In this section, we list some details of the dynamic model of the hydraulic system.

\section{A. Upstream Boundary Condition}

The upstream boundary condition at the junction of the tank and the feedline consists of a time-dependent total pressure $P_{t}$ constructed by adding the tank ullage pressure $p_{u}$ (taken to be constant in the examples presented here) and the acceleration head of the liquid in the tank:

$$
P_{t}=p_{u}-\rho a_{t} h_{t}
$$

where $\rho$ is the propellant density (assumed constant and uniform in the tank), $a_{t}$ is the acceleration of the tank, and $h_{t}$ is the difference in the $x$ coordinates of the junction and the free surface of the tank. All of these quantities may be functions of time. In addition, the static pressure at the junction $p_{t}$ is calculated as

$$
p_{t}=P_{t}-\frac{1}{2} \rho u_{t}^{2}
$$

where $u_{t}$ is the volumetric velocity at the junction.

\section{B. Feedlines}

The relative motion of the liquid at any point in the suction line (and relative to the suction line) is governed by the following basic continuity and momentum equations for compressible, frictional flow in a duct:

$$
\begin{gathered}
\frac{\partial(\rho A)}{\partial t}+\frac{\partial(\rho u A)}{\partial x}=0 \\
\rho\left[\frac{\partial u}{\partial t}+u \frac{\partial u}{\partial x}\right]=-\frac{\partial p}{\partial x}-\rho a-\rho \omega^{2} x-2 \rho \omega u-\rho \frac{\partial \omega}{\partial t} x-\frac{\rho f u|u|}{2 D}
\end{gathered}
$$

where $D$ and $f$ are the diameter and the friction factor of the duct. The set of equations for the compressible flow are closed through the definition of the speed of sound $c$ in the duct derived from the Joukowsky equation (2):

$$
\frac{\partial(\rho A)}{\partial p}=\frac{A}{c^{2}}
$$

Since these frictionally modified Euler equations apply in an accelerating coordinate system, the second, third, fourth, and fifth terms in the momentum equation [Eq. (7)] must be included in the formulation. These additional, pseudopressure terms (see [22]), which reduce to zero in the absence of acceleration, involve not only the relative velocity $u$ and the acceleration $a$ but also the rate of rotation $\omega$ of the coordinate system about the axes perpendicular to the flow direction. However, in the examples presented in this paper, the rate of rotation $\omega$ is taken to be zero. Similar equations are used for the discharge line downstream of the pump.

\section{Valves}

Valves and orifices are modeled using a simple lumped parameter loss coefficient, so that the properties upstream and downstream of the restriction (denoted, respectively, by subscripts 1 and 2) are related by

$$
\begin{gathered}
Q_{2}=Q_{1} \\
p_{2}-p_{1}=-\frac{1}{2} k \rho\left(\frac{Q_{1}}{A_{1}}\right)^{2}-L \frac{\mathrm{d} Q_{1}}{\mathrm{~d} t}-\rho a\left(x_{2}-x_{1}\right)
\end{gathered}
$$

where $k$ and $L$ are the loss coefficient and the inertance of the component. Here again, the pseudopressure effects are included through the inclusion of the terms involving the acceleration $a$.

\section{Accumulator}

The accumulator is modeled as a capacitor with an initial pressure $p_{c}$. The pressure difference between the suction line and the inside of the accumulator, $p-p_{c}$, should satisfy

$$
\begin{gathered}
Q_{2}+Q_{c}=Q_{1} \\
p-p_{c}=\frac{1}{C_{c}} \int_{0}^{t} Q_{c} \mathrm{~d} t+R_{c} Q_{c}+L_{c} \frac{\mathrm{d} Q_{c}}{\mathrm{~d} t}-\rho a h_{c}
\end{gathered}
$$

where $p_{c}$ is the initial accumulator gas pressure; $Q_{c}$ is the flow rate into the accumulator; $R_{c}, L_{c}$, and $C_{c}$ are the resistance, inertance, and compliance of the accumulator; and $h_{c}$ is the elevation difference between the suction line next to the accumulator and the free surface in the accumulator.

\section{E. Cavitating Pump}

The flow rates $Q$ relative to the pump and the total pressures $P$ at the inlet to (subscript 1) and discharge from (subscript 2) the pump are related by the conventional pump transfer matrix $[\underline{1}, \underline{11}, \underline{13}]$

$$
\begin{gathered}
P_{2}=\left(m_{p}+1\right) P_{1}+\Delta H\left(\Omega, Q_{1}\right) \\
-L_{p} \frac{\mathrm{d} Q_{1}}{\mathrm{~d} t}-\rho a\left[x_{2}-\left(m_{p}+1\right) x_{1}\right] \\
Q_{2}=Q_{1}-C_{p} \frac{\mathrm{d} P_{1}}{\mathrm{~d} t}-M_{p} \frac{\mathrm{d} Q_{1}}{\mathrm{~d} t}
\end{gathered}
$$

where, as is conventional, $\left(m_{p}+1\right)$ is called the pump gain; $\Delta H\left(\Omega, Q_{1}\right)$ represents the static pump total pressure increase (which normally has a negative slope and is often linearized as $R_{p} Q_{1}$, where $R_{p}$ is called the pump resistance); $L_{p}$ is the pump inertance; $C_{p}$ is called the cavitation compliance; and $M_{p}$ is called the mass flow gain factor. Potential values of these dynamic pump characteristics have been the subject of much discussion and research $[1,11,13]$ and, following this brief summary of the hydraulic models, we include comments on the values for these characteristics in the next section.

\section{F. Downstream Boundary Condition}

The downstream boundary condition is provided by the total pressure $P_{\mathrm{cc}}$ in the combustion chamber or the catchment tank into 


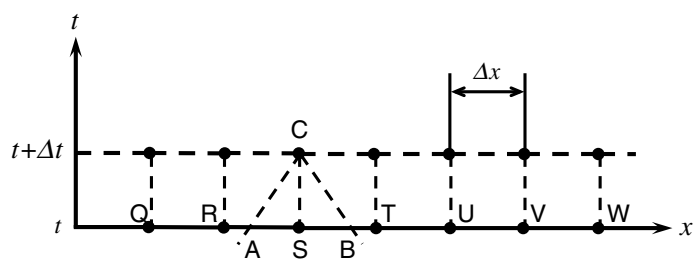

Fig. 5 Example of numerical solution by method of characteristics.

which the liquid discharges (this may be a function of time). Since the liquid typically discharges through a large resistance (a valve, orifice, or injector), the static pressure $p_{e}$ at the end of the line downstream of the pump (at $x=x_{e}$ ) should satisfy a relation similar to Eq. (10), namely,

$$
P_{\mathrm{cc}}-p_{e}=-\frac{1}{2} k_{e} \rho\left(\frac{Q_{e}}{A_{e}}\right)^{2}-\rho a\left(x_{\mathrm{cc}}-x_{e}\right)
$$

where $Q_{e}$ and $A_{e}$ are the flow rate and duct area at the end of the discharge line, and $k_{e}$ is the loss coefficient in that terminating resistance.

The equations described previously were solved numerically by standard methods traditionally used for fluid transients [1,2]. Equations (6-8) for compressible flow in a duct are solved using the method of characteristics, and the boundary conditions between components are solved numerically using the Newton-Raphson method. We will briefly review these methods here.

The typical numerical solutions by the method of characteristics are depicted graphically in Fig. $\underline{5}$. By assuming the constant density of liquid $\rho$, speed of sound $c$, and the uniform pipe, so that $f$ is constant, Eqs. (6- $-\underline{8}$ ) yield the following set of two ordinary differential equations:

1) In a frame of reference moving with velocity $u+c$,

$$
\frac{\mathrm{d} u}{\mathrm{~d} t}+\frac{1}{\rho c} \frac{\mathrm{d} p}{\mathrm{~d} t}+\frac{f u|u|}{2 D}-\frac{u a}{c}=0
$$

2) In a frame of reference moving with velocity $u-c$,

$$
\frac{\mathrm{d} u}{\mathrm{~d} t}-\frac{1}{\rho c} \frac{\mathrm{d} p}{\mathrm{~d} t}+\frac{f u|u|}{2 D}+\frac{u a}{c}=0
$$

In the finite difference forms, Eqs. (16) and (17) become

1) On the characteristic line $\mathrm{d} x / \mathrm{d} t=u+c$ (upstream characteristic line),

$$
\frac{u_{C}-u_{A}}{\Delta t}+\frac{1}{\rho c} \frac{p_{C}-p_{A}}{\Delta t}+\frac{f u_{A}\left|u_{A}\right|}{2 D}-\frac{u_{A} a_{A}}{c}=0
$$

2) On the characteristic line $\mathrm{d} x / \mathrm{d} t=u-c$ (downstream characteristic line),

$$
\frac{u_{C}-u_{B}}{\Delta t}-\frac{1}{\rho c} \frac{p_{C}-p_{B}}{\Delta t}+\frac{f u_{B}\left|u_{B}\right|}{2 D}+\frac{u_{B} a_{B}}{c}=0
$$

Here, $u_{A}, u_{B}, p_{A}$, and $p_{B}$ are velocities and pressures at points $A$ and $B$ at time $t$, which are determined by interpolation between the known values at points such as $R, S$, and $T$ in Fig. 5. By solving the simultaneous first-order equations (18) and (19), $u_{C}$ and $p_{C}$, the velocity and pressure of the position $\bar{C}$ at time $\bar{t}+\Delta t$, are obtained. Similarly, the upstream and downstream properties of the component that lies between the pipe $\left(u_{1}, u_{2}, p_{1}\right.$, and $\left.p_{2}\right)$ are obtained using the Newton-Raphson method to solve the four simultaneous equations: the characteristic equation (18) of the upstream line, a set of two equations that relate the upstream and downstream properties of each component listed previously but in the finite difference forms, and the characteristic equation (19) of the downstream pipe.

\section{Dynamic Pump Characteristics}

Since pump cavitation and its dynamic characteristics play a substantial role in determining the response of the hydraulic system, it is important to use values for the dynamic pump characteristics that are as accurate as possible. Hence, we digress here to add further comment on these parameters of the problem. In the absence of cavitation, and neglecting the compressibility of the liquid and structure of the pump, it follows from fluid continuity that $m_{p}, C_{p}$, and $M_{p}$ should be equal to zero. It also follows that, at low rates of change, it is also reasonable to set $R_{p}$ equal to the slope of the static pump performance curve, as described by Brennen [1]. Moreover, reasonably accurate values of the pump inertance $L_{p}$ can be obtained from first principles. With these assumptions, it transpires that, under stable, noncavitating conditions, the pump resistance and inertance simply and benignly add to the resistance and inertance of the feedlines and invoke no surprise in the response of the hydraulic systems. In this scenario, one complication that we do not include in the present calculations is the dependence of the pump resistance and inertance on the oscillation frequency (or rate of change of the flow variables). It may be important to note that these frequency variations can be quite substantial. For example, Rubin [13] notes that the pump resistance $R_{p}$ may increase substantially with frequency, while the inertance $L_{p}$ may decrease.

However, pump cavitation changes that conclusion and, as Rubin [13] demonstrated, radically alters the response. All of the pump dynamic characteristics become functions of the extent of the cavitation in the pump, as well as the other mean operating conditions. The extent of the cavitation is conveniently characterized by the cavitation number $\sigma$, defined as

$$
\sigma=\frac{p_{1}-p_{v}}{(1 / 2) \rho u_{\text {tip }}^{2}}
$$

where $p_{v}$ is the saturated vapor pressure, and $u_{\text {tip }}$ is the velocity of the inducer tip. The pattern and extent of cavitation also change with the operating condition (represented most conveniently by a flow coefficient), but we shall simplify this presentation by confining the calculations to the design flow coefficient. We focus, therefore, on the effects of $\sigma$ on the pump dynamic characteristics, particularly the pump compliance $C_{p}$, mass flow gain factor $M_{p}$, and the consequent effects on the system response. We therefore digress further to comment on the appropriate values of $C_{p}$ and $M_{p}$ for the LE-7A turbopump. In this regard, we are primarily guided by the available experimental measurements for this and other turbopumps [11-13]. However, since the LE-7A turbopump includes an inducer that displays a cavitation pattern that is somewhat different from the SSME inducer of [11], the following procedure was conducted in an attempt to extract some confirmational data from the LE-7A turbopump ground tests.

During the static ground tests of this turbopump, naturally occurring pressure fluctuations and structural oscillations were monitored. Moreover, pressures were measured at several locations along the suction and discharge lines so that fluctuating flow rates could also be calculated. From these measurements, it was then possible to estimate pertinent pump compliance $C_{p}$ and mass flow gain factor $M_{p}$ values, and to do so for a range of cavitation numbers. For a variety of reasons (principally the uncertainty in the calculation of the instantaneous flow rate difference between the suction and the discharge), these calculations are subject to large uncertainties (about an order of magnitude), but we present the results here, since they provide some comparisons with the much more accurate values of these quantities obtained by imposed fluctuation of the hydraulic system [11]. The values obtained from the static ground-based tests on the LE-7A turbopump are shown in Fig. 6, where they are compared with the more accurate data from [11]. We note that the present estimates of the LE-7A compliance are quite consistent with those of $[11,12]$. They are also consistent with the compliance value derived from a simple model of the peak natural vibration frequency observed during the turbopump ground tests with an accumulator (the second configuration). That calculation assumes a mode involving the inertance of the suction line between the accumulator and the inducer and derives a nondimensional inducer cavitation compliance of 0.6 at a cavitation number of $\sigma=0.04$. Figure $\underline{6}$ exhibits $M_{p}$ values for the LE-7A that are less than the values from 


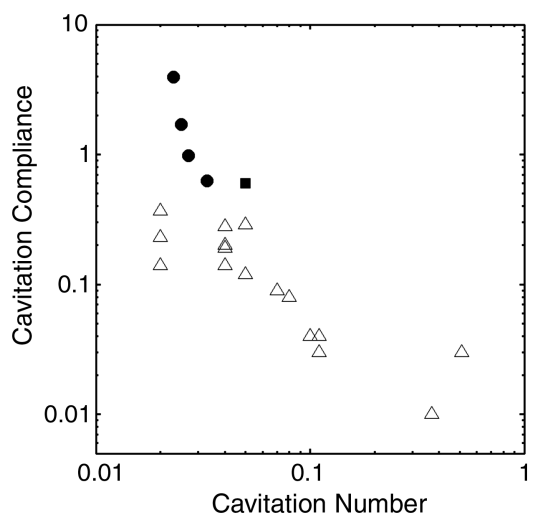

a)

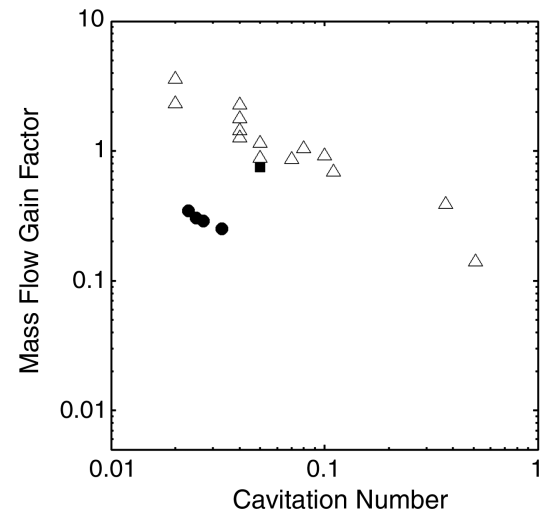

b)

Fig. 6 Pump dynamic characteristics as a function of cavitation number: nondimensional a) cavitation compliance, $C_{p} \rho u_{\mathrm{tip}}^{2} Z_{p} / 2 \pi A_{p} D_{p}$, and b) mass flow gain factor, $M_{p} u_{\text {tip }} Z_{p} / \pi D_{p}$. Values for LE-7A turbopump (solid circles) are compared with SSME impeller measurements (open triangles) [11] and LE-7 measurements (solid squares) [1]․

[11], although the difference is still within the uncertainty of the present calculations. We do note, however, that the LE-7A design involves smaller angles of incidence and, therefore, less backflow cavitation than the SSME design. Therefore, a lower value for $M_{p}$ would not be unexpected. The validity and potential uncertainties in the dynamic pump coefficients have recently been examined by Rubin [13].

For the purposes of the present comparisons, the LE-7A estimates for $C_{p}$ and $M_{p}$ as functions of $\sigma$ that are presented in Fig. $\underline{6}$ were applied to the calculations of the responses in each of the four hydraulic system configurations. In addition, a value of unity was assumed for the pump gain $\left(m_{p}=0\right)$, although we again note that $[\underline{11}, \underline{13}]$ suggest a value that increases with frequency.

\section{Dynamic Responses in Ground-Based Configurations}

Calculations for the various configurations of pumping or propulsion systems described previously were made for a range of frequencies $f$, made nondimensional by dividing by the pump rotating frequency. These results were then compared with the corresponding test data from experiments on the liquid oxygen pump in the Japanese LE-7A rocket engine. It is important to emphasize that, although these configurations correspond to different test facilities, the pump being tested is the same in all configurations. Thus, in each of the models, we use the same static pump performance curves and the same sets of pump transfer matrices; only the facility properties differ in each of the configurations. In addition, in the three groundtest configurations, low-level white noise (random pressure perturbations) is generated and injected at the pump inlet in order to provide a trigger for potential cavitation surge, should that be inclined to occur. The same level of noise is used in all the calculations. This technique is based on the assumption that the cavitation surge (and other dynamic responses) observed in the ground-based tests is triggered by random pressure perturbations generated within the turbopump itself. We now describe the results obtained for each of the configurations.

\section{A. Cold-Test Facility Incorporating a Turbopump Without Accumulator}

The calculated results for the cold-test facility for the turbopump without an accumulator are shown in Fig. 7. For a cavitation number greater than 0.04 , Fig. 7 shows that the inducer pressure fluctuations are very small. However, when the cavitation number is decreased into the range 0.033 to 0.020 , pressure fluctuations at a nondimensional frequency of $f=0.22$ become dominant. This resonant frequency, $f=0.22$, corresponds to the third organ-pipe mode of oscillation of the compressible liquid in the suction line, with the open end at the tank and the closed end at the pump. However, the maximum pressure amplitude that occurs at the inducer discharge is less than $0.4 \%$ of the inducer tip dynamic pressure. The pressure amplitude at the pump discharge (not shown) is very small because of the large resistance and the inertance of the pump and the discharge line. Note that small pressure fluctuations with the nondimensional frequency of 0.04 and 0.13 are also observed at pump inlet and inducer discharge. These frequencies correspond to the first and the second organ-pipe modes of the suction line.

The corresponding data from the measurements taken during the turbopump tests in the first configuration are presented in Fig. 8. As in the calculations, the pressure oscillations exhibit a resonance at $f=0.22$ and onset when the cavitation number decreases into the range 0.033 to 0.020 . But the amplitudes are less than $0.3 \%$ of the

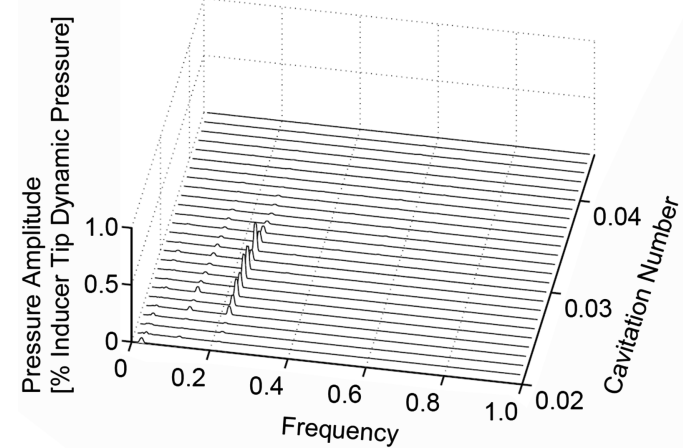

a)

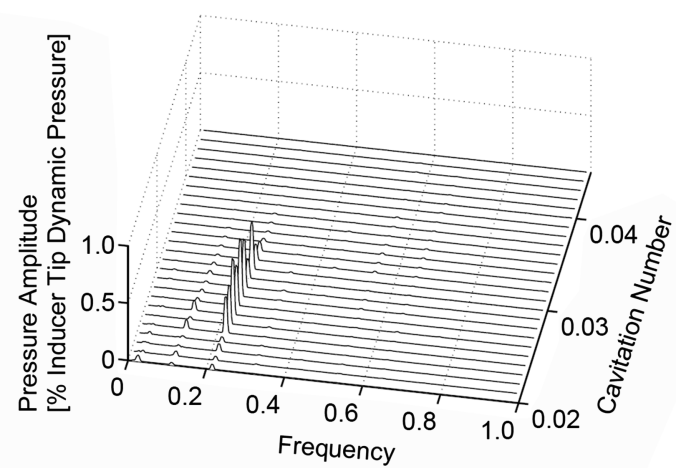

b)

Fig. 7 Model calculations for inducer a) inlet pressure and b) discharge pressure in cold-test facility without accumulator, first configuration. 


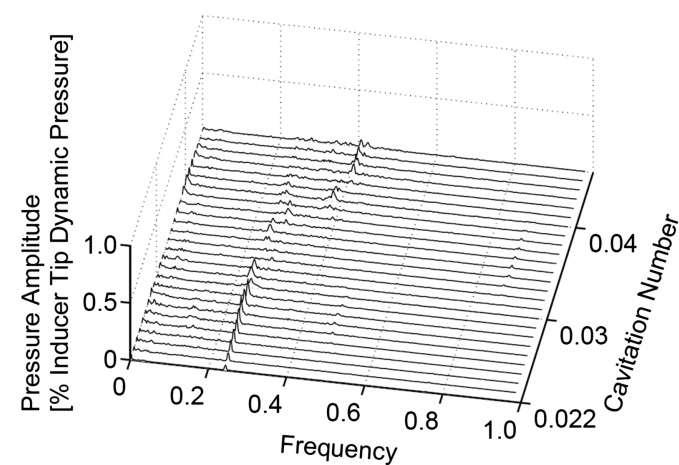

a)

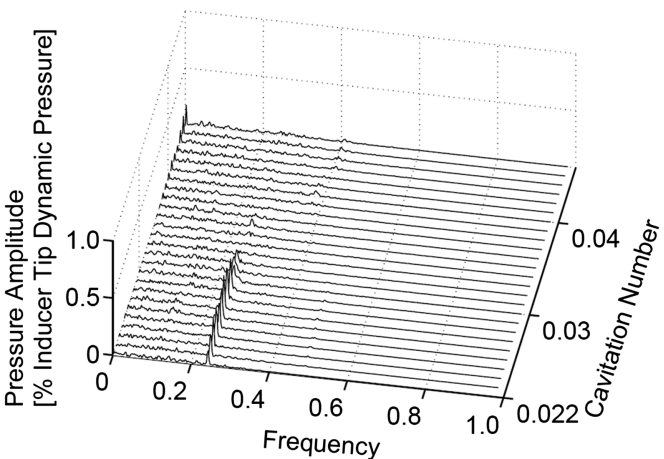

b)

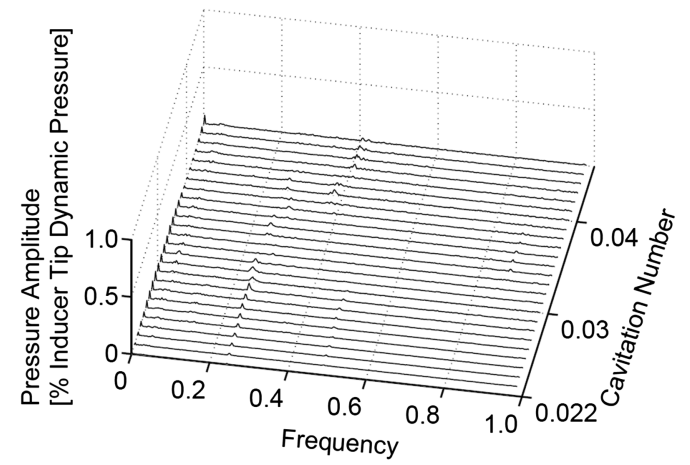

c)

Fig. 8 Measurements from cold-test facility without accumulator of inducer a) inlet pressure and b) discharge pressure to be compared with Fig. 7. Also shown is c) main impeller discharge pressure.

inducer tip dynamic pressure and are therefore not consequential. Thus, the model accurately reproduces the test data, both in frequency and amplitude.

Parenthetically, we also note that the Helmholtz frequency $f_{s}$ of the incompressible liquid in the suction line between the tank and the pump is given by

$$
f_{s}=\frac{\left(L_{s} C_{p}\right)^{-1 / 2}}{2 \pi \Omega}
$$

where $L_{s}$ is the inertance of the whole suction line. This Helmholtz frequency that has a nondimensional value of about $f_{s}=0.02$ does not clearly present, both in the model results and test data. This is the result of large inertance of the suction line.

\section{B. Cold-Test Facility Incorporating a Turbopump} With Accumulator

The calculated model results for the cold-test facility with the accumulator (the second configuration) are shown in Fig. 9. The most obvious change is the appearance of a natural resonant oscillation of the flow between the accumulator and the cavitation in the pump. This occurs because of the short length (and therefore small inertance) of fluid between the accumulator and the cavitation. As the cavitation number decreases and the cavitation compliance increases, the frequency of this natural cavitation surge decreases. The nondimensional frequency $f_{\text {sc }}$ follows the Helmholtz frequency calculated by Eq. (21) but with $L_{s}$ replaced by $L_{\mathrm{sc}}$, the inertance of the liquid in the short column between the accumulator and the pump. For $\sigma>0.040$, the inducer pressure fluctuations involved are very small. But when $\sigma$ is reduced to $0.037, f_{\text {sc }}$ becomes about 0.22 and therefore matches the third organ-pipe mode between the tank and the pump. This double resonance results in a sudden, substantial increase in the magnitude of the pressure oscillations. Moreover, with further decrease in $\sigma$ to 0.035 , the fluctuation magnitude decreases again as the double resonance has passed. Despite the double resonance, the largest pressure amplitudes are less than $1 \%$ of the inducer tip dynamic pressure. As in the first configuration, this magnitude may be inconsequential.

The corresponding experimental measurements are presented in Fig. 10 and show good qualitative agreement with the model. As in

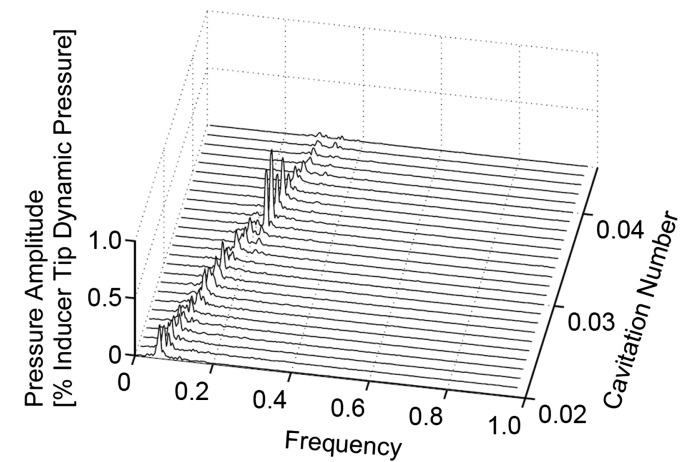

a)

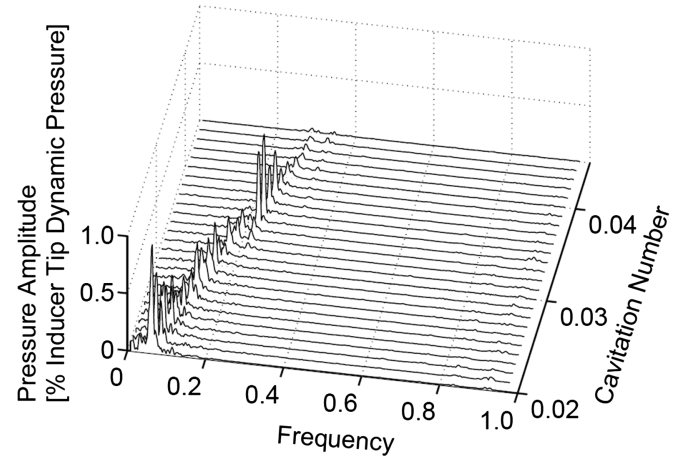

b)

Fig. 9 Model calculations for inducer a) inlet pressure and b) discharge pressure in cold-test facility with accumulator, second configuration. 


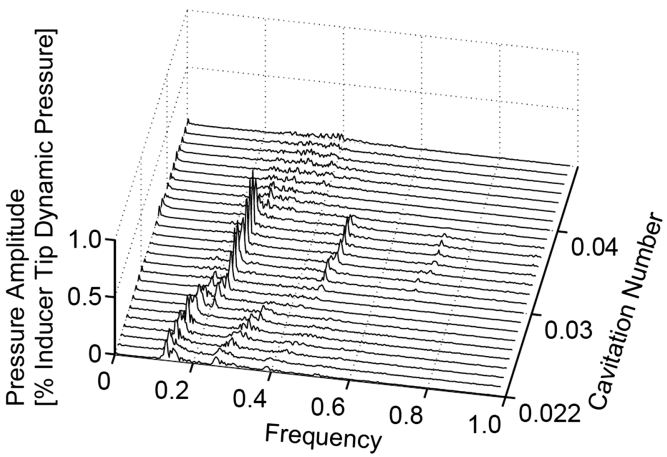

a)

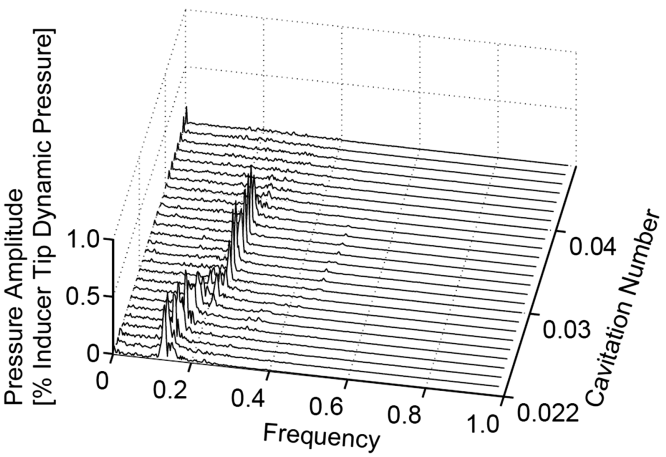

b)

Fig. 10 Measurements from cold-test facility with accumulator of inducer a) inlet pressure and b) discharge pressure to be compared with Fig. 9 .

the first configuration without the accumulator, the maximum pressure amplitude occurs at the inducer discharge, while the pressure amplitudes at the pump discharge are much smaller because of the large resistance and the inertance of the pump and the discharge line. Thus, the model reproduces the test data accurately, both in the frequency and the amplitude. However, the higher harmonics observed on the test do not appear in the model calculations, probably because the model does not include adequate nonlinear effects. We also note that the natural frequency $f_{\mathrm{sc}}$ is slightly lower in the model calculation, especially at low cavitation numbers. This is probably caused by an estimated cavitation compliance, which is slightly larger than in reality.

\section{Hot-Firing Engine Test Facility}

The model results for the third configuration, the hot-firing engine test, are presented in Fig. 11. As in the second configuration, the response is dominated by a strong resonance of the suction line between the pump and the accumulator. The nondimensional frequency of this resonance decreases from 0.5 to 0.3 as the cavitation number is decreased from 0.05 to 0.02 . The frequencies are higher than in the second configuration, because the accumulator is much closer to the turbopump; hence, $L_{\mathrm{sc}}$ is smaller. However, the pressure amplitudes are still small: less than $0.01 \%$ of the inducer tip dynamic pressure, even at the inducer discharge. Even though the suction line organ-pipe frequencies exist at every 0.02 step in the nondimensional frequency spectrum, no large pressure oscillation magnitudes occur, because the suction line in the hot-firing engine test facility is very long and the suction line resistance is large. The corresponding measurements from the hot-firing test are presented in Fig. 12. Again, the model results appear to simulate the test data very well, matching both the frequency and the amplitude. The major difference between Figs. 11 and 12 is that the results of the model calculations appear noisy compared with the experimental data. The reason is that almost all the organ-pipe modes around the natural frequency respond in the model calculations while they are muted in

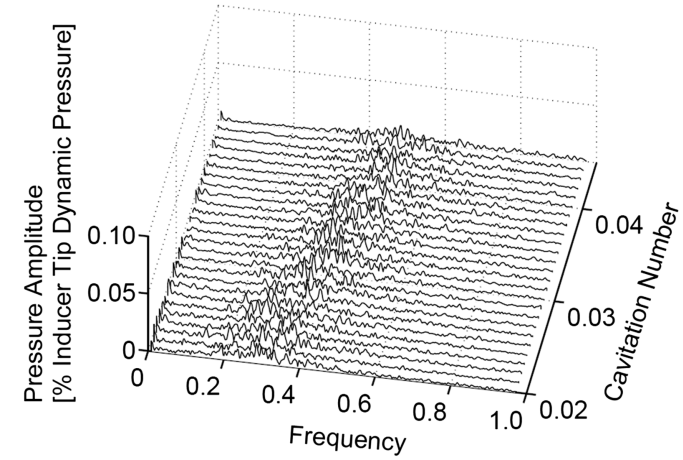

a)

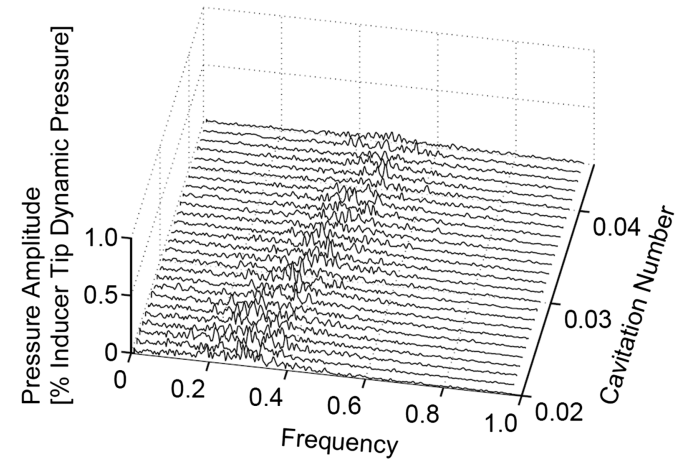

b)

Fig. 11 Model calculations for inducer a) inlet pressure and b) discharge pressure in hot-firing engine test, third configuration.

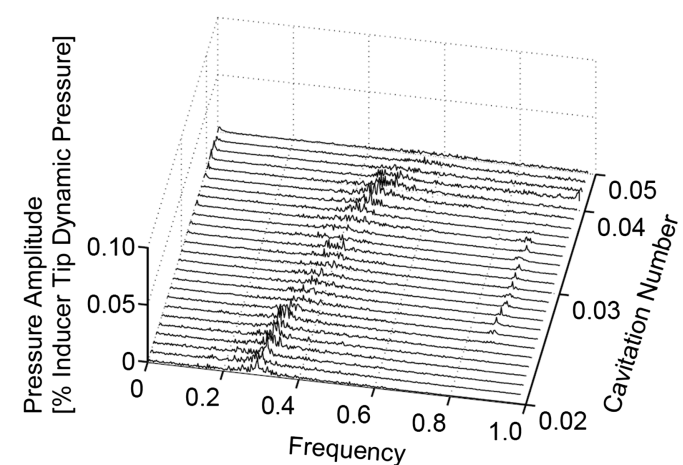

a)

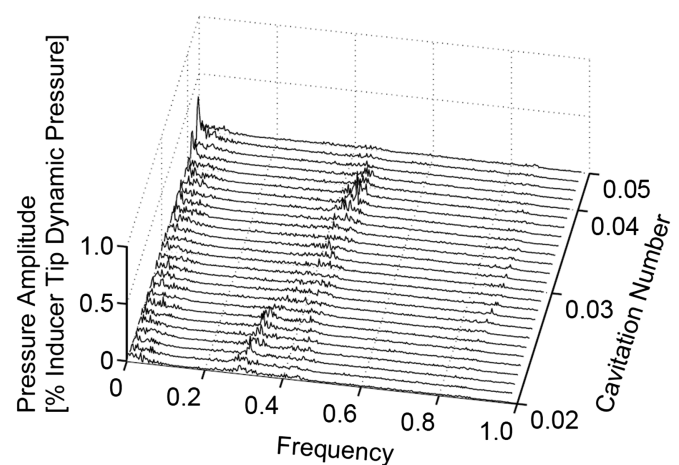

b)

Fig. 12 Measurements from hot-firing engine test of inducer a) inlet pressure and b) discharge pressure to be compared with Fig. 11. 


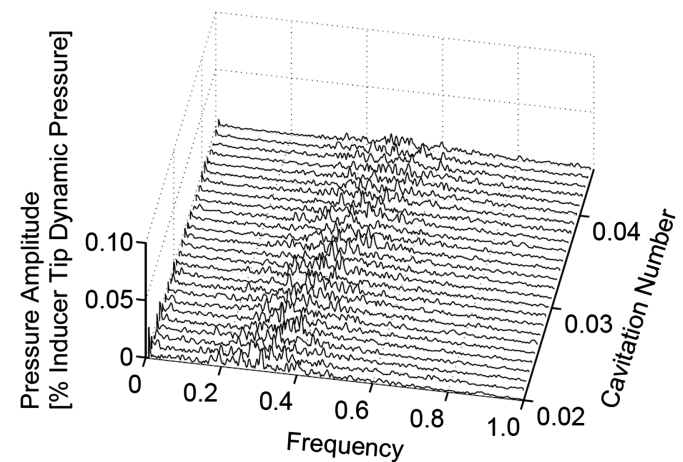

a)

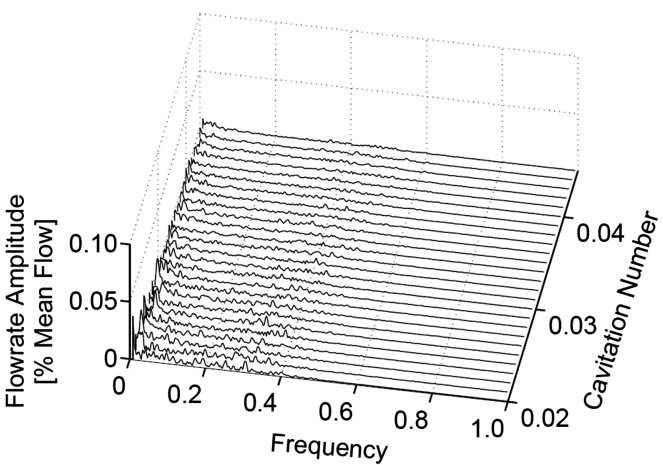

b)

Fig. 13 Model calculations for flight configuration in a static, nonaccelerating frame: a) turbopump inlet pressure and b) turbopump discharge flow rate.

the tests because, in the test facility, the actual suction line has a complex geometry that inhibits the standing wave development.

These comparisons between the model predictions and the ground-based test stand measurements provide considerable confidence in the predictive capability of the model. Thus, we move, in the next section, to examine the model predictions for the actual rocketbased propulsion system. The preceding model calculations and ground-based tests also demonstrate that the hydraulic system is very stable within a nonaccelerating environment.

\section{Dynamic Response in Accelerating Frame}

We now turn to the fourth configuration: the hydraulic propulsion system in a vehicle that is experiencing oscillations. First, however, we examine the response of the flight configuration in a static, nonaccelerating frame, but with the same white noise excitation used in the first three configurations. The results of this preliminary test are presented in Fig. 13, where it is seen that only very low-level oscillations are generated: namely, pressure oscillations less than $0.01 \%$ of inducer tip dynamic pressure and flow rate oscillations less than $0.01 \%$ of mean flow. Thus, as we might have anticipated, like the first three configurations, the flight configuration is very stable in a nonaccelerating frame.

Then, the model was used to examine the response in a sinusoidally accelerating frame with an acceleration amplitude of $0.1 \mathrm{~m} / \mathrm{s}^{2}$ at various nondimensional frequencies ranging from 0 to 0.5 . This magnitude would be characteristic of the background excitation experienced in the rocket environment. For example, a thrust oscillation with an amplitude of $0.5 \%$ (of the total thrust) will generate an oscillating acceleration of $0.1 \mathrm{~m} / \mathrm{s}^{2}$ of the center of mass of the whole vehicle accelerating at $19.6 \mathrm{~m} / \mathrm{s}^{2}(2 \mathrm{G})$. The model results under noncavitating conditions are shown in Fig. 14, which plots the response of the pressures and the flow rates at tank outlet, inducer inlet, and pump discharge as a function of the excitation frequency. The conclusion is that, in the absence of cavitation, the system response is quite muted with pressure oscillation magnitudes less than $0.05 \%$ of inducer tip dynamic pressure and flow rate oscillation magnitudes less than $0.02 \%$ of mean flow. The pressure magnitudes in the suction line exhibit almost no frequency dependence, while the pump discharge pressure exhibits a minimum at $f=0.05$, which corresponds to the line resonance of the compressible liquid between the accumulator and the combustion chamber. The tank outlet flow rate exhibits peaks at each of the resonant frequencies of the organ-pipe modes of the compressible liquid between the tank and the accumulator, with the maximum flow rates occurring at the inlet of the accumulator. Meanwhile, the pump inlet flow rate decreases almost monotonically with frequency as a result of the line resistances. The pump discharge flow rate into the combustion chamber decreases with frequency, exhibiting a maximum of about $0.01 \%$ of mean flow rate at the lowest frequencies and a decrease at higher frequencies. But, in summary, in the absence of cavitation, the flight system behaves like a continuous compressible tube and the pressure oscillation amplitudes do not substantially exceed the quasi-static acceleration head response at the low frequency given by $\rho a h$, where $h$ is the elevation difference between the two ends.

Finally, we present the key result of this paper: namely, the response of the flight hardware to the same range of global oscillation (an acceleration magnitude of $0.1 \mathrm{~m} / \mathrm{s}^{2}$ for a range of oscillation frequencies) when the pump is cavitating. Figure 15 presents the results for the lowest cavitation number examined: namely, $\sigma=0.02$. Compared with the results presented in Fig. 14, it is clear that the result is a violent resonant response with amplitudes about two orders of magnitude greater than in the absence of cavitation. The

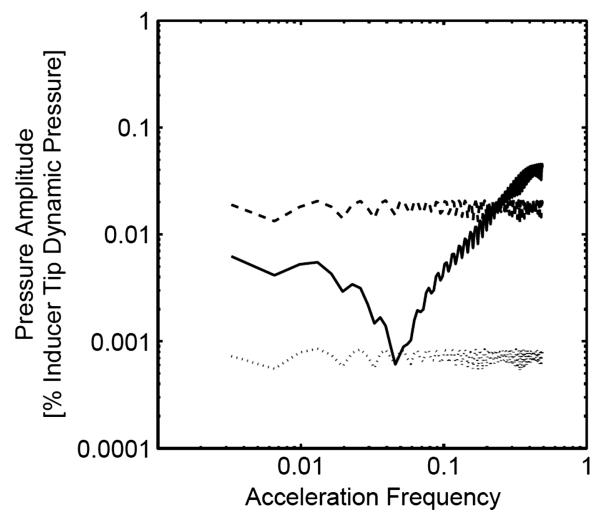

a)

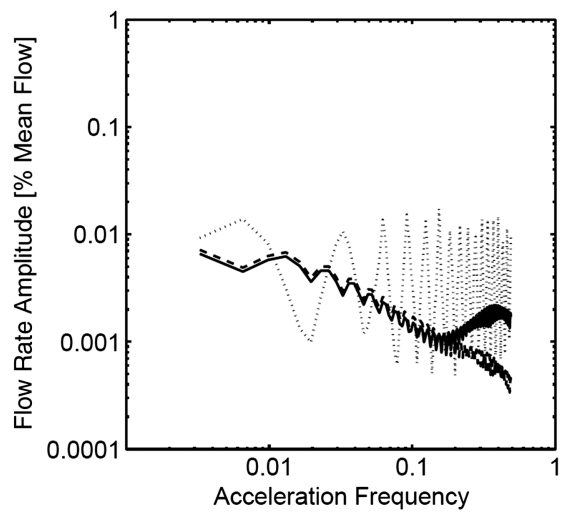

b)

Fig. 14 Model calculations of frequency response of flight configuration to global oscillation with magnitude of $0.1 \mathrm{~m} / \mathrm{s}^{2}$ in absence of pump cavitation: a) pressure amplitudes and b) flow rate amplitudes. Solid, dashed, and dotted lines, respectively, represent main impeller discharge, inducer inlet, and tank outlet quantities. 


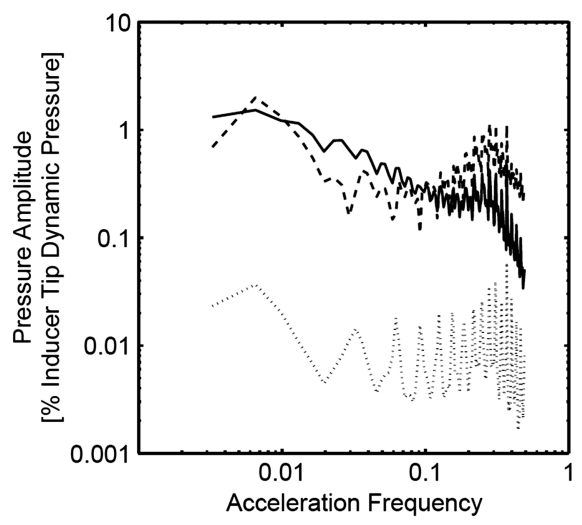

a)

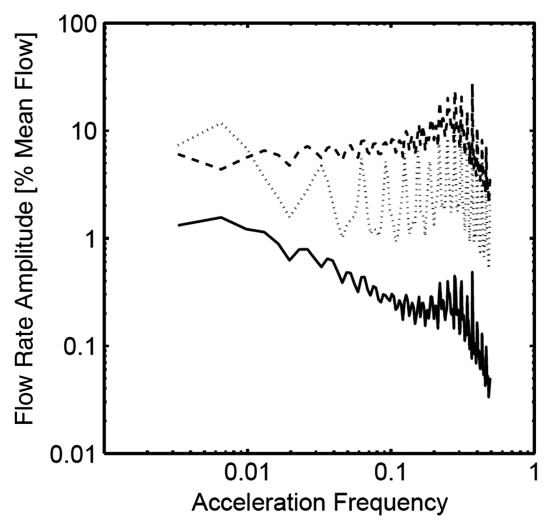

b)

Fig. 15 Model calculations of frequency response of flight figuration to global oscillation with magnitude of $0.1 \mathrm{~m} / \mathrm{s}^{2}$ when pump cavitation number is $\sigma=0.02$ : a) pressure amplitudes and b) flow rate amplitudes. Solid, dashed, and dotted lines, respectively, represent main impeller discharge, inducer inlet, and tank outlet quantities.

pressure oscillation magnitudes are more than $2 \%$ of the inducer tip dynamic pressure, and the flow rate oscillation magnitudes are more than $20 \%$ of the mean flow. Under these cavitating conditions, the largest flow rate magnitudes occur between the accumulator and the inducer at all frequencies and the largest pressure amplitudes occur at the inducer discharge. Thus, the flow rate oscillation between the accumulator and the inducer dominates the overall response and excites the rest of the system like an oscillating piston. The suction line from the tank to the accumulator also plays a role, albeit a secondary role. When the frequency of the "piston" coincides with an organ-pipe mode of the compressible liquid between the tank and the cavitating inducer, the entire system exhibits a peak response, and this happens at each of those organ-pipe modes. There is also an important global response maximum near the natural cavitation surge frequency of 0.3 ; at higher frequencies, the response dies off rather rapidly. We also note one mitigating factor, namely, that the pump discharge flow rate oscillations are generally smaller than those upstream of the pump because of the large resistance and inertance of the pump itself. Other cavitation numbers follow a similar pattern with the same natural peak frequencies and amplitudes in the same proportions, as indicated in Fig. 13.

Thus, the model calculations demonstrate how a violent resonant response can occur in the accelerating flight environment when pump cavitation is present and that this response can occur even when all the ground tests (and the model flight calculations without cavitation) indicate a stable and well-behaved response. The difficulty of duplicating these adverse flight environments in any ground test (and, therefore, of examining such an adverse condition) makes accurate model calculations an almost essential design tool.

\section{Conclusions}

A time-domain response analysis model has been developed to evaluate the dynamic response of pumping systems in an accelerating environment. The particular application considered concerns the stability of liquid pumping systems in rockets, but findings could apply to a wide range of other contexts in which pumping systems may be subject to shaking. The paper particularly focuses on the role cavitation may play in determining the dynamic response and demonstrates the important role cavitation can play in altering the response. The dynamic model is first verified by comparing the results with experimental measurements made during ground-based tests of the Japanese LE-7A rocket engine. These tests were conducted in three different test facilities and involved various levels of pump cavitation, including cases leading to natural cavitation surge. By using the models to evaluate the response of these systems, it is shown that various inlet and discharge line resonances occur and that features such as cavitation or the incorporation of an accumulator alter these resonances. As in the experiments, the analyses demonstrated that the LE-7A propulsion system was very stable in a nonaccelerating frame; even at the resonance corresponding to the frequency of cavitation surge, the pressure oscillations were minimal (less than $0.01 \%$ of the inducer tip dynamic pressure). The model was also used to examine the response in an accelerating frame; sinusoidal oscillations of the vehicle over a range of frequencies were explored. Under noncavitating conditions, a vehicle acceleration of $0.1 \mathrm{~m} / \mathrm{s}^{2}$ produced pump inlet pressure oscillations of less than $0.05 \%$ and pump discharge flow rate oscillations of less than $0.02 \%$. On the other hand, under cavitating conditions at $\sigma=0.02$, a vehicle acceleration of $0.1 \mathrm{~m} / \mathrm{s}^{2}$ produced inducer inlet flow rate oscillations of more than $20 \%$ and discharge flow rate oscillations of $2 \%$. The obvious conclusion is that ground-based tests may not adequately predict the potential resonances of the pumping system, especially when the pump is cavitating. Even if the pumping system is stable in the static analysis and in ground-based tests conducted in a static test facility, oscillations of the whole vehicle can cause a substantial amplitude of flow oscillation, particularly when the pump is cavitating.

\section{Acknowledgments}

The first author wishes to acknowledge the support of Makiko Hori, during his visit to the California Institute of Technology, and the Japan Aerospace Exploration Agency.

\section{References}

[1] Brennen, C. E., Hydrodynamics of Pumps, Oxford Univ. Press, New York, 1994, pp. 1-316.

[2] Wylie, E. B., Streeter, V. L., and Suo, L., Fluid Transients in Systems, Prentice-Hall, Upper Saddle River, NJ, 1993, pp. 1-463.

[3] Greitzer, E. M., "The Stability of Pumping Systems: The 1980 Freeman Scholar Lecture," Journal of Fluids Engineering, Vol. 103, No. 2, Jun. 1981, pp. 193-242. doi: $10.1115 / 1.3241725$

[4] Rubin, S., "Longitudinal Instability of Liquid Rockets Due to Propulsion Feedback (POGO)," Journal of Spacecraft and Rockets, Vol. 3, No. 8, 1966, pp. 1188-1195. doi: $10.2514 / 3.28626$

[5] Rubin, S., "Prevention of Coupled Structure-Propulsion Instability (POGO)," NASA SP-8055, Oct. 1970.

[6] Oppenheim, B. W., and Rubin, S., "Advanced Pogo Stability Analysis for Liquid Rockets," Journal of Spacecraft and Rockets, Vol. 30, No. 3, 1993, pp. 360-373. doi: $10.2514 / 3.25524$

[7] Dotson, K. W., Rubin, S., and Sako, B. H., "Mission-Specific Pogo Stability Analysis with Correlated Pump Parameters," Journal of Propulsion and Power, Vol. 21, No. 4, 2005, pp. 619-626. doi:10.2514/1.9440

[8] Ohashi, H., "Analytical and Experimental Study of Dynamic Characteristics of Turbopumps," NASA TN D-4298, 1968.

[9] Brennen, C., and Acosta, A. J., "The Dynamic Transfer Function for a Cavitating Inducer," Journal of Fluids Engineering, Vol. 98, No. 2, 1976, pp. 182-191. doi: $10.1115 / 1.3448255$ 
[10] Ng, S. L., and Brennen, C. E., "Experiments on the Dynamic Behavior of Cavitating Pumps," Journal of Fluids Engineering, Vol. 100, No. 2, June 1978, pp. 166-176 doi: $10.1115 / 1.3448625$

[11] Brennen, C. E., Meissner, C., Lo, E. Y., and Hoffman, G. S., "Scale Effects in the Dynamic Transfer Functions for Cavitating Inducers," Journal of Fluids Engineering, Vol. 104, No. 4, 1982, pp. 428-433. doi:10.1115/1.3241875

[12] Shimura, T., "Geometry-Effects in the Dynamic Response of Cavitating LE-7 Liquid-Oxygen Pump," Journal of Propulsion and Power, Vol. 11, No. 2, 1995, pp. 330-336. doi: $10.2514 / 3.51429$

[13] Rubin, S., "An Interpretation of Transfer Function Data for a Cavitating Pump," 40th AIAA/ASME/SAE/ASEE Joint Propulsion Conference, Fort Lauderdale, FL, AIAA Paper 2004-4025, July 2004.

[14] Cervone, A., Tsujimoto, Y., and Kawata, Y., "Evaluation of the Dynamic Transfer Matrix of Cavitating Inducers by Means of a Simplified 'Lumped-Parameter' Model,' Journal of Fluids Engineering, Vol. 131, No. 4, 2009. doi:10.1115/1.3089535

[15] Gibbs, K. P., and Oliver, A. G., "Special Requirements for Pumps for Sodium Cooled Fast Reactors," Pumps for Nuclear Plant Convention, Univ. of Bath, Bath, England, U.K., April 1973, pp. 119-124.

[16] Braisted, D. M., and Brennen, C. E., "Auto-Oscillation of Cavitating Inducers," Polyphase Flow and Transport Technology, American Soc. of Mechanical Engineers, New York, 1980, pp. 157-166.
[17] Tsujimoto, Y., Kamijo, K., and Yoshida, Y., "A Theoretical Analysis of Rotating Cavitation in Inducers," Journal of Fluids Engineering, Vol. 115, No. 1, March 1993, pp. 135-141. doi:10.1115/1.2910095

[18] Kamijo, K., Shimura, T., and Tsujimoto, Y., "Experimental and Analytical Study of Rotating Cavitation," ASME Cavitation and GasLiquid Flow in Fluid Machinery and Devices, Vol. 190, American Soc. of Mechanical Engineers, Fairfield, NJ, 1994, pp. 33-43.

[19] Hashimoto, T., Yoshida, M., Watanabe, M., Kamijo, K., and Tsujimoto, Y., "Experimental Study on Rotating Cavitation of Rocket Propellant Pump Inducers," Journal of Propulsion and Power, Vol. 13, No. 4, 1997, pp. 488-494. doi: $10.2514 / 2.5210$

[20] Zoldaz, T., "Observations on Rotating Cavitation and Cavitation Surge from the Development of the Fastrac Engine Turbopump," 36th AIAA/ ASME/SAE/ASEE Joint Propulsion Conference, Huntsville, AL, AIAA Paper 2000-3403, July 2000.

[21] Yoshida, Y., Sasao, Y., and Watanabe, M., "Thermodynamic Effect on Rotating Cavitation in an Inducer," Journal of Fluids Engineering, Vol. 131, No. 9, Sept. 2009, Paper 091302. doi:10.1115/1.3192135

[22] Batchelor, G. K., An Introduction to Fluid Dynamics, Cambridge Univ. Press, New York, 1967, pp. 1-615.

M. Costello Associate Editor 